\title{
Bündnis Zukunft der Industrie - Industriepolitische Herausforderungen und Perspektiven aus Sicht der IG Metall
}

\author{
WOLFGANG LEMB
}

Der industrielle Sektor war und ist das Fundament für Wachstum, Wohlstand und gute Arbeit in Deutschland. Die exportorientierte Industrie bildet nach wie vor das Rückgrat der deutschen Wirtschaftskraft. Die Industriegüterproduktion hat einen Anteil von etwa $40 \%$ am gesamtwirtschaftlichen Produktionswert der deutschen Wirtschaft. Zusammen mit den produktionsnahen Dienstleistungen hängen sogar knapp $60 \%$ direkt oder indirekt von der industriellen Produktion ab. Die industrielle Wertschöpfung ist damit für den Wohlstand und die politische Stabilität in Deutschland von größter Bedeutung.

Nicht umsonst gilt der noch vor wenigen Jahren in der Kritik stehende - weil an der „Old Economy“ festhaltende - deutsche Industriestandort heute als Vorbild. Eine wesentliche Erklärung, wie die deutsche Ökonomie vom „kranken Mann Europas“ zum Stabilitätsanker mutierte, liegt in der Stärke der Industrie. Deutschland ist unter den reifen OECDÖkonomien die einzige Nation, die seit 1995 ihren Industrieanteil am Bruttosozialprodukt bei $22 \%$ halten konnte. Ein Wert, von dem andere europäische Staaten nur träumen können. Deutschland nimmt damit inzwischen für die Industrien in den europäischen Nachbarländern eine zentrale Drehscheibenfunktion wahr. Die Finanz- und Wirtschaftskrise der Jahre 2008 und 2009 hat gezeigt, dass Deutschland durch kooperatives Vorgehen der Sozialpartner, erfolgreiche Konjunkturpolitik und abgestimmte Arbeitsmarktpolitik weit besser durch die Krise gekommen ist als andere vergleichbare Länder. Der Anspruch auf gelebte Mitbestimmung gilt aber nicht nur für Krisenzeiten.

\section{Industriepolitische Perspektive gesucht}

Demografischer Wandel, Globalisierung, Digitalisierung, Klimawandel und die Notwendigkeit, Ressourcen effizienter zu nutzen sowie eine nachhaltige Energiewende voranzutreiben, sind Megatrends, die ein koordiniertes Vorgehen zwischen
Gewerkschaften, Unternehmen und Staat notwendig machen. Deshalb hat die IG Metall mit dem Bundesverband der Deutschen Industrie (BDI) die Initiative ergriffen und gemeinsam mit dem Bundeswirtschaftsministerium im März 2015 das Bündnis „Zukunft der Industrie“ gegründet. Es ist als Gesprächsplattform konzipiert und soll bewirken, dass Deutschland auch im 21. Jahrhundert ein erfolgreiches Industrieland bleibt. Dabei sollen die industriepolitischen Kompetenzen der mittlerweile 15 Bündnispartner gebündelt, Netzwerke geschaffen und ausgebaut werden, um die Industrie modern und zukunftsfähig zu gestalten.

Ziel des Bündnisses ist der Erhalt und die offensive Weiterentwicklung des Industriestandortes Deutschland. In fünf Arbeitsgruppen wird seit Juni 2015 an den Themen Akzeptanz und Attraktivität der Industrie, investitionsstarke Industrie, Zukunft der Arbeit in Industrie und industrienahen Dienstleistungen sowie Wertschöpfungsstrukturen der Zukunft und internationale Wettbewerbsfähigkeit gearbeitet. Zudem werden die Rahmenbedingungen für sichere Arbeitsplätze, gute Arbeit, Wohlstand und Fortschritt diskutiert.

\section{Wertschöpfungsketten erhalten und ausbauen}

Die deutsche Industrie ist stark internationalisiert. Die Globalisierung und die tiefe Einbindung in die internationale Arbeitsteilung führen zu tief greifenden Veränderungen und neuen Herausforderungen: Absatzmärkte verschieben sich, neue Werke entstehen künftig in den Wachstumsmärkten. Auch die Beschäftigtenstruktur verändert sich: der Anteil der Produktionsarbeit verringert sich, Forschung und Entwicklung sowie industrielle Dienstleistungen nehmen zu. Der Fachkräftebedarf ist in vielen Bereichen mittlerweile signifikant.

Hinzu kommt der technologische Wandel, beispielsweise bei Antriebssystemen, Leichtbau- techniken und der Digitalisierung der Produktion. Dieser besitzt nicht nur eine technische $\mathrm{Di}$ mension, sondern Wertschöpfungsketten werden neu sortiert. Die Arbeitsabläufe und damit die Anforderungen an menschliche Arbeit verändern sich in kurzen Intervallen.

Die Bundesrepublik verfügt an vielen Stellen noch über fast vollständige Wertschöpfungsketten, wo Hersteller und Zulieferer eng zusammenarbeiten. Das haben wir einem dichten Netz von Grundstoffindustrien bis hin zu spezialisierten Zulieferern und großen Systemanbietern auf wachstumsstarken Leitmärkten zu verdanken. Doch durch den Globalisierungsdruck besteht die Gefahr, dass sich diese Wertschöpfungsketten auflösen.

Die Wertschöpfungsketten müssen also insgesamt betrachtet und umfassende Strategien zu ihrem Erhalt und Ausbau entworfen werden. Dies wird uns nur gelingen, wenn wir eine Investitionsund Innovationsoffensive zugunsten der deutschen Industrie erreichen. Dazu brauchen wir - eine Harmonisierung zentraler Standortbedingungen (z. B. Unternehmenssteuern) auf Ebene der EU und nachgeordneter Ebene sowie eine Eindämmung des Subventionswettbewerbs - eine stärkere makroökonomische Koordinierung mit Berücksichtigung ihrer Auswirkungen auf Branchen und Regionen

- den Ausbau der Tarifbindung in Deutschland sowie die Förderung von Tarif- bzw. Kollektivverhandlungen an ausländischen Standorten deutscher Unternehmen

- faire Handelsabkommen, für die Investitionsschutz und Schiedsgerichte in entwickelten Rechtssystemen verzichtbar sind und die ILOMindeststandards akzeptieren.

\section{Beschäftigte in den Mittelpunkt stellen}

Wenn wir auch in Zukunft eine leistungsstarke, international wettbewerbsfähige und innovative Industrie halten wollen, müssen wir nicht nur 
mehr in innovative Technologien und Infrastruktur investieren, sondern vor allem in die Kompetenzen und die Entwicklung der Beschäftigten. Sie sind der Motor von Innovationen, sie erarbeiten reale Werte, sichern das wirtschaftliche Wachstum und damit den Wohlstand unserer Gesellschaft. Die nachhaltige Sicherung von qualifizierten Fachkräften ist also eine der zentralen Herausforderungen der nächsten Jahre.

Schon heute werden vielerorts Fachkräfte gesucht, sowohl im Handwerk als auch in der Metall- und Elektroindustrie. Mit dem demografischen Wandel nehmen diese Probleme weiter zu: Dem Arbeitsmarkt stehen in Zukunft mehr ältere und weniger jüngere Menschen zur Verfügung. Das hat eine Reihe von Konsequenzen für die betriebliche Ebene, für Aus- und Weiterbildung. Auf den Stärken des dualen Systems können wir dabei aufbauen. Gute Bildung und eine bessere Qualifikation der Beschäftigten, gerade was die MINT-Berufe (Mathematik, Informatik, Naturwissenschaften, Technik) angeht, stehen ganz oben auf der Agenda. Da kann man nicht alles dem Staat überlassen, sondern die Unternehmen selbst müssen wesentlich mehr in Bildung investieren und sich um einen ausgewogenen Mix aus Hochschulabsolventen und dual Ausgebildeten bemühen, wenn sie wettbewerbsfähig bleiben wollen. Mit der tariflichen Bildungsteilzeit haben die Tarifparteien insbesondere die Herausforderung angenommen, auch die heutigen Belegschaften stärker an den Veränderungs- und Innovationsprozessen zu beteiligen und ihnen mit einer besseren Verzahnung von Arbeits- und Lebenswelten eine langfristige Perspektive zu bieten. Denn auch das stärkt den Industriestandort Deutschland für die Zukunft.

\section{Chancen für eine „Gute Industriepolitik“}

Damit die Industrie der Zukunft ein starker Kern der deutschen Wirtschaft bleiben kann, muss die Industriepolitik angesichts der strukturellen Veränderungsprozesse neu ausgestaltet werden. Dies kann nur im Dialog zwischen allen relevanten Partnern funktionieren. Wir sehen darin eine historische Chance, dass es nach Jahrzehnten einer quasi religiösen Marktgläubigkeit nun eine Renaissance der Industriepolitik gibt. Die IG Metall will dabei mitbestimmen und die Bedingungen im Sinne der Belegschaften gestalten. Wenn durch die Digitalisierung monotone Arbeiten wegfallen und anspruchsvollere Tätigkeiten entstehen, dann zeigt das auch, was in Zukunft not- wendig sein wird: neue Qualifikationsstrategien, die den Beschäftigten dabei helfen, neue und bessere Aufgabenfelder zu übernehmen. Wir wollen, dass die Industrie die Herausforderung der digitalisierten Wirtschaft meistert, indem sie auf selbstverantwortliches Arbeiten, eine lernförderliche Arbeitsorganisation und dezentrale Assistenzsysteme setzt.

Die gemeinsame Initiative für eine dynamische und innovative Industrie zielt deshalb darauf $a b$, Beschäftigung zu sichern und neue Beschäftigungschancen zu nutzen. Denn die Stärke und damit auch die Zukunft der deutschen Industrie liegen in herausragenden, innovativen Qualitätsprodukten, die von motivierten, qualifizierten Beschäftigten und Fachkräften in fairer Arbeit zu gesicherten Tarifbedingungen produziert werden.

Lange fehlte auf der politischen Seite der Partner, mit dem man gemeinsam Kernfragen einer nachhaltigen Industriepolitik angehen konnte. Damit sollen keineswegs Interessenunterschiede verwischt werden. Vielmehr geht es um eine prinzipielle Dialogbereitschaft und die Suche nach Schnittmengen für ein gemeinsames industriepolitisches Vorgehen. Gute Industriepolitik kann nach unserer Auffassung nur mit Mitbestimmung und der Einbindung von Betriebsräten und Gewerkschaften funktionieren. Gerade in Zeiten des Wandels ist es notwendig, die Interessen der Belegschaften in die betriebliche und in die öffentliche Diskussion einzubringen.

Anders als ihre Vorgängerinnen hat die Große Koalition den Wert der Industrie für die wirtschaftliche Entwicklung Deutschlands erkannt und bekennt sich zu einer aktiven Industriepolitik, die über das Setzen von Rahmenbedingungen deutlich hinausgeht. Insofern ist Bewegung in die bundesdeutsche Industriepolitik gekommen. Im Rahmen der verschiedenen Initiativen wurde ein umfassender Dialogprozess auf den Weg gebracht, an dem die Gewerkschaften von Anfang an beteiligt sind.

Dadurch wird ein Klima der Kooperation geschaffen, womit die Gefahren eines unvollständigen Wissens über zukünftige industrielle Entwicklungen vermindert sowie die Fehler eines einseitigen Top-down-Ansatzes vermieden werden können. Allerdings macht ein anderer Stil noch keine wirklich neue Industriepolitik aus. Die Zukunft wird zeigen, ob der neue Anlauf wirklich zu konkreten Maßnahmen, Initiativen und Projekten führt, die den Ansprüchen der IG Metall an eine „Gute Industriepolitik“ gerecht werden.

\section{Industrieakzeptanz erhöhen}

Jedoch: Es geht nicht nur um die richtigen Konzepte, sondern auch darum, wie sie vermittelt werden. Und die Akzeptanz von neuen, industriellen Großvorhaben stößt in der Bevölkerung auf teilweise erhebliche Skepsis und sogar offenen Widerstand (Stromtrassen, Stuttgart 21 etc.). Umfragen zeigen aber auch, dass es in der deutschen Bevölkerung keine grundsätzliche Ablehnung von industrieller Wertschöpfung und neuen Technologien gibt. Gerade bei Alltags- und Gebrauchstechnologien (Kommunikation, EDV, Haushaltsgeräte) gibt es eine große Aufgeschlossenheit für Innovationen. Wenig hilfreich ist die Stimmungsmache von Politikern, die nach dem „St.-Florians-Prinzip“ beispielsweise den für die Energieversorgung der Zukunft notwendigen Ausbau der Netzinfrastruktur behindern und nebenbei noch versuchen, die Vorbehalte von Bürgerinnen und Bürgern gegen die Energiewende zu richten.

Gewerkschaften und Betriebsräte stehen in diesen Prozessen und Diskussionen häufig vor einer widersprüchlichen Situation. Auf der einen Seite treten sie als Beschäftigtenvertreter in den betroffenen Unternehmen, als Unterstützer von Industrie, Technologien, industrieller und technologischer Entwicklung sowie von Infrastrukturmaßnahmen auf. Auf der anderen Seite sind sie nicht selten im Zuge eines zivilgesellschaftlichen Engagements als Akteure in Bündnissen und Bürgerinitiativen gegen solche Maßnahmen aktiv.

Wir wollen mit dem Bündnis „Zukunft der Industrie" auch einen neuen gesellschaftlichen Konsens für die Modernisierung und den Ausbau der Infrastruktur herstellen sowie, damit verbunden, eine umfassende und frühzeitige Bürgerbeteiligung bei der Planung neuer Infrastrukturvorhaben gewährleisten. Dazu gehört die Einsicht, dass Technologien und industrielle Produktion nie völlig ohne Risiken sein können, auch nicht in der Produktion und dem Vertrieb regenerativer Energien.

\section{Wie geht es weiter?}

Auch weil es der erklärte Wille der Beteiligten ist, auf konsensuale Art und Weise zu konkreten Ergebnissen zu kommen, um die politischen Entscheidungen vorzubereiten, dürfen wir keine Schnellschüsse erwarten. Vielmehr geht es erst einmal darum, anzuerkennen, dass ein Umdenken in Gang gekommen ist. Der eingeschlagene Weg weist zum richtigen Ziel. Insbesondere in der 
zuletzt hinzugekommenen Arbeitsgruppe „Internationale Wettbewerbsfähigkeit der Industrie“ werden aber die nach wie vor von unterschiedlichen Interessen bestimmten Positionen bei der Beurteilung politischer Entscheidungen zu Freihandel und supranationaler Regulierung aufeinandertreffen. Das wurde schon in den Branchendialogen Maschinenbau und Elektroindustrie deutlich: Während gemeinsam die Bedeutung von Innovationen und Marktführerschaft bei Energieeffizienzlösungen betont werden, liegen die Posi- tionen bei der Frage des Abbaus von Handelshemmnissen unverändert weit auseinander.

Wenn wir aber die Bedingungen für mehr Wachstum und Beschäftigung verbessern wollen, dann müssen sich die Bündnispartner auf jene Themen konzentrieren, bei denen sie inhaltlich übereinstimmen. Nur wenn wir uns auf klare Spielregeln und Strukturen verständigen und alles vermeiden, was zu einer Politik der wechselseitigen Überforderung führt, werden wir gemeinsam erfolgreich sein.

\section{AUTOR}

WOLFGANG LEMB, geschäftsführendes Vorstandsmitglied der IG Metall, verantwortlich für die Bereiche Internationales und Europa, Industrie-, Struktur- und Energiepolitik sowie das IG-Metall Projekt "Zukunft Ost”.

@ wolfgang.lemb@igmetall.de 